\title{
Transverse Myelitis
}

National Institute of Neurological Disorders and Stroke (NINDS)

\section{Source}

National Institute of Neurological Disorders and Stroke (NINDS). Transverse Myelitis

Information Page.

Transverse myelitis is an inflammation of the spinal cord. The inflammation interrupts communications between nerve fibers in the spinal cord and the rest of the body, affectng sensation and nerve signaling below the damage to the protective nerve coating called myelin. The segment of the spinal cord at which the damage occurs determines which parts of the body are affected. Damage at one segment will affect function at that level and below. In people with transverse myelitis, the myelin damage most often occurs in nerves in the upper back, causing problems with leg movement and bowel and bladder control, which require signals from the lower segments of the spinal cord.

Symptoms may develop suddenly or over days or weeks. Classic symptoms include:

- weakness of the arms and legs,

- sensory alterations such as burning, pricking, numbness, or sensory loss,

- pain (usually in the lower back or down the legs and arms or around the torso),

- and bowel and bladder dysfunction.

Some cases of transverse myelitis occur on their own, but a number of conditions appear to cause the disorder, including immune system disorders, bacteial and viral infections, fungal infections, and various parasites. Transverse myelitis can affect people of any age, but a peak in incidence rates appears to occur between 10 and 19 years of age and 30 to 39 years of age. 